\section{SCIENCE CHINA \\ Physics, Mechanics \& Astronomy}

-Editorial•

Editor's Focus
March 2020 Vol. 63 No. 3: 230361

https://doi.org/10.1007/s11433-019-1491-1

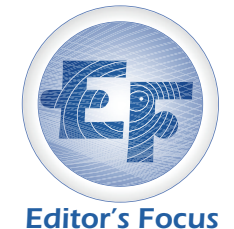

\title{
Quantum secure direct communication with an untrusted Charlie using imperfect measurement devices
}

Quantum secure direct communication (QSDC) is a branch of quantum communication that transmits messages directly, rather than via a cryptographic key, in the quantum form [1-3]. As communication security becomes ever important today, the early use of quantum-proof cryptography is considered capable of minimizing the future exposure [4]. While quantum key distribution is regarded as such an approach, the potential of QSDC is also worth noting [4], and its rapid development has been witnessed in recent years [5-10].

In practice, the devices used for quantum communication are yet imperfect, and an eavesdropper may seize the opportunity to steal information without being detected. One of the biggest defects is in the measurement device. To solve this problem, instead of perfecting these devices, which is not available in a short term, one can design protocols to circumvent the existing defects. This is realized by introducing an untrusted third party to perform the measurement using imperfect devices. This technique was originally designed for quantum key distribution. In a recent study [11], Zhou et al. from Tsinghua University, Nanjing University of Posts and Telecommunications, and Southampton University designed a measurement-device-independent (MDI) QSDC protocol.

It is not easy to design a MDI-QSDC protocol because of the higher security requirement in QSDC. Actually, the MDI-QSDC protocol proposed in ref. [11] is the MDI-version of the single-photon based DL04 QSDC protocol presented in ref. [3]. The essential process is a variant of quantum teleportation. Alice prepares a sequence of EPR-pairs, and Bob prepares a sequence of single photons. Then Alice sends a sequence of photons, each from an EPR pair, to an untrusted Charlie, and Bob sends his single photon sequence to Charlie, too. Then Charlie makes Bell-basis measurement to each pair he receives, and publishes the results. This is equivalent to the teleportation of Bob's single photon sequence to Alice, but with the Bell-basis measurement performed by Charlie. Then Alice encodes her message on the remaining single photon sequence in her hand, whose quantum states are teleported from Bob, and sends the encoded photons to Charlie again, who performs single qubit measurements and announces the results. In this way, Charlie performs all the Bell-basis and single qubit measurements. He must conduct the measurements honestly, because any of his attempts to sabotage the process will be detected by Alice and Bob. This is done by inserting some checking single photons in Alice's sequences and Bob's sequence. The MDI-versions of the EPR-based QSDC protocols have also been constructed using the idea presented here. They are given in ref. [12].

XinCheng Xie

School of Physics, Peking University, Beijing 100871, China

1 G. L. Long, and X. S. Liu, Phys. Rev. A 65, 032302 (2002).

2 F. G. Deng, G. L. Long, and X. S. Liu, Phys. Rev. A 68, 042317 (2003).

3 F. G. Deng, and G. L. Long, Phys. Rev. A 69, 052319 (2004).

4 E. B. Kania, Mil. Cyber Aff. 3, 10 (2018).

5 J. Y. Hu, B. Yu, M. Y. Jing, L. T. Xiao, S. T. Jia, G. Q. Qin, and G. L. Long, Light Sci. Appl. 5, e16144 (2016).

6 W. Zhang, D. S. Ding, Y. B. Sheng, L. Zhou, B. S. Shi, and G. C. Guo, Phys. Rev. Lett. 118, 220501 (2017).

7 F. Zhu, W. Zhang, Y. Sheng, and Y. Huang, Sci. Bull. 62, 1519
(2017).

8 R. Qi, Z. Sun, Z. Lin, P. Niu, W. Hao, L. Song, Q. Huang, J. Gao, L. Yin, and G. L. Long, Light Sci. Appl. 8, 22 (2019).

9 S. S. Chen, L. Zhou, W. Zhong, and Y. B. Sheng, Sci. China-Phys. Mech. Astron. 61, 090312 (2018).

10 L. Zhou, Y. B. Sheng, and G. L. Long, Sci. Bull. (2019).

11 Z. R. Zhou, Y. B. Sheng, P. H. Niu, L. G. Yin, G. L. Long, and L. Hanzo, Sci. China-Phys. Mech. Astron. 63, 230362 (2020).

12 P. H. Niu, Z. R. Zhou, Z. S. Lin, Y. B. Sheng, L. G. Yin, and G. L. Long, Sci. Bull. 63, 1345 (2018). 